\title{
Gestión de políticas de sostenibilidad en organizaciones académicas: El caso de la Universidad de Extremadura
}

\author{
Rafael Robina-Ramírez \\ Universidad de Extremadura \\ rrobina@unex.es \\ Libertad Moreno Luna \\ Universidad de Extremadura \\ limorenol@alumnos.unex.es
}

Resumen: El objetivo de esta investigación es conocer las actuales políticas de sostenibilidad en la Universidad y proponer un nuevo modelo que contribuya a aumentar la conciencia medioambiental de los alumnos de la universidad las áreas de economía y empresa. Para desarrollar la conciencia medioambiental entre estudiantes (DCM) se han determinado 3 factores a partir de un proceso de análisis y validación de variables entre estudiantes universitarios. Estos factores son: la Formación de profesores en sostenibilidad (FPS), actividades de concienciación medioambiental $(A C M)$ e incorporación de competencias medioambientales transversales (CMT). La metodología aplicada para analizar el modelo estructural fue el Modelo de Ecuación Estructural (SEM) a través del paquete informático SmartPls-3. La muestra estaba formada por 420 estudiantes pertenecientes a las Facultades de Económicas y la Facultad de Empresa, Finanzas y Turismo de la Universidad de Extremadura. Los resultados de la encuesta arrojan datos que justifican la introducción de políticas de sostenibilidad en la Universidad tanto a nivel profesorado como alumno. Dichas politicas deberían resaltar la importancia de dotar de contenido a la competencia transversal dirigida a la protección medioambiental entre los futuros empresarios de nuestro país.

Palabras clave: sostenibilidad; estudiantes; Universidad; empresas; Extremadura 


\begin{abstract}
The aim of this research is to study the management of sustainability policies in the University of Extremadura and to identify the most important factors in developing the environmental awareness of students of business, finance, economics and tourism. Three factors have been analyzed within the framework of sustainability policies in the academic field: teacher training, environmental awareness activities and transversal environmental competences. The methodology used was the Structural Equation Model of the SmartPls-3 software package. The sample consisted of 420 students belonging to the Faculties of Economics, Business, Finance and Tourism of the Universidad de Extremadura. The survey found that selected constructs influenced the main objective of the University's sustainability policies, which is to increase environmental awareness among its students. The study particularly highlights the importance of the transversal competence, which seeks to promote environmental protection among future entrepreneurs in Spain.
\end{abstract}

Keywords: Sustainability; students; University; companies; Extremadura 


\section{Introducción}

De acuerdo con la UNESCO (2010) la era industrial moderna es responsable de la contaminación ambiental de la superficie y la atmósfera de la Tierra. Esta devastadora transformación contribuye a una creciente escasez de agua y recursos en el planeta (ONU, 2018), llegando a hoy en día a ser un problema prioritario (UNESCO, 2015).

El énfasis de las organizaciones internacionales sobre el deterioro medioambiental (ONU, 2015) ha provocado, entre otras medidas, que el sector empresarial y académico se hayan unido en la búsqueda de soluciones el insostenible modelo de producción y consumo actual (AACSB International, 2015). Este modelo devastador de desarrollo económico y medioambiental ha conducido a una situación crítica de la que no será fácil salir (Colín, 2003).

Como contrapartida, se han propuesto medidas de desarrollo sostenible tanto por parte de académicos y responsables de la formulación de políticas (UN, 2012; Beland Lindahl, 2016; Ull, et alii, 2010; Robina, 2016) desde la perspectiva ambiental, económica y social (Giddings et alii, 2002). La educación ha sido uno de los elementos clave para mejorar dicho desarrollo sostenible (Vare y Scott, 2007).

En el ámbito educativo, el desarrollo sostenible se ha identificado como la preservación de los recursos naturales (perspectiva ambiental) en cooperación con las comunidades que residen alrededor de dichos recursos (perspectiva socioeconómica) (Rauch, 2002; Giddings et alii, 2002; Ramírez, Seeliger y Di Prieto, 2016). Sin embargo, todavía no se han encontrado evidencias sobre el papel transformador de la educación medioambiental entre aquellas comunidades, especialmente entre las generaciones futuras (Cicmil, Ecclestone y Collins, 2007).

El cambio hacia un consumo más sostenible sólo puede lograrse a través de un trabajo educativo continuo, tanto en las escuelas y universidades como en las empresas (Colín, 2003; Landrum y Edwards, 2011; UNESCO, 1997; Zealand, 2004; Robina, Merodio y McCallum, 2020) y esto requiere mejoras significativas en la forma en que pensamos y actuamos (UNESCO, 2014). Esta actitud responsable hacia el medioambiente se ha introducido en el ámbito académico con el objetivo de lograr una sociedad medioambientalmente sostenible (UNECE, 2005; UNESCO, 2005, 2010, 2014).

El ámbito de la universidad, donde se promueve la investigación y el desarrollo de la cultura (Aznar et alii, 2014) la enseñanza de valores medioambientales y actitudes en defensa de la naturaleza es de vital importancia (Cincera y Krajhanzl, 2013). 
Las experiencias en ámbitos educativos basadas en planes de estudio y en la mejora del conocimiento medioambiental entre estudiantes ha tenido un efecto limitado en la transformación de las actitudes de los estudiantes (Olsson et alii, 2016; Boeve-de Pauw y Van Petegem, 2011, People and Work Unit, 2007; Robina-Ramírez y Medina-Merodio, 2019). En concreto, en las facultades de empresa y finanzas la educación medioambiental no ha conseguido integrar todavía la sostenibilidad en los programas de estudio universitarios (Hart, 2009; Landrum y Edwards, 2011).

Sin embargo, solo en algunos países desarrollados con alta conciencia del daño de la falta de concienciación sostenible han comenzado a apostar por un sistema educativo en el que se incluyen materias relacionadas con el respeto al medio ambiente (Tilbury, 2011).

Con el objetivo de introducir nuevas políticas de sostenibilidad en la Universidad en las áreas de economía y empresa el estudio pretende responder a la pregunta: ¿qué factores educativos permiten mejorar las actitudes de los estudiantes universitarios hacia el medioambiente?

La novedad del artículo se centra en la propuesta un modelo de desarrollo de la concienciación medioambiental entre estudiantes universitarios como guía de las políticas de sostenibilidad en la Universidad. A partir de la metodología SEM-PLS diversas relaciones de causalidad entre variables han sido propuestas con implicaciones teóricas y prácticas tanto para la investigación explicativa (confirmatoria) como para la predictiva (exploratoria) de fenómenos sociales como la enseñanza de sostenibilidad (Henseler, Hubona y Ray, 2016; Hair et alii, 2017).

\section{La enseñanza de sostenibilidad ambiental en las Universidades}

En las últimas décadas, la enseñanza de sostenibilidad en España ha sido escasa, más basada en la conservación de los recursos naturales para protegerlos del agresivo crecimiento económico en las últimas décadas (Gutiérrez, Benayas y Calvo, 2006). Así lo recoge expresamente el Libro blanco de la educación ambiental en España (Calvo y Corrales, 1999, p. 23) expresando la necesidad de un cambio en la mentalidad de los productores y empresarios que gestionan dichos recursos.

Este lento proceso de cambio de la conservación y protección hacia la enseñanza del desarrollo sostenible pretende integrar el desarrollo sostenible en todo el sistema educativo universitario (UNESCO, 2014).

A consecuencia de este cambio de mentalidad un escaso número de universidades empezaron a embarcarse en adoptar un comportamiento más responsable 
(Waas et alii, 2010) firmando declaraciones nacionales e internacionales en defensa de prácticas sostenibles a través de la docencia, investigación y divulgación (Lozano et alii, 2013; Wright, 2002, 2004; Waas et alii, 2010). En el ámbito universitario, e aumento en la concienciación medioambiental podía venir introducido a través de tres pilares básicos: docencia, la investigación y la vida universitaria Capdevila (1999) o bien en la mejora de la gestión de los aspectos ambientales en los edificios y en el campus, gestión y minimización de residuos, movilidad y transporte o también a través de una cuidada comunicación y sensibilización ambiental (Solís, 2008).

En España, debido al diferente posicionamiento ante la sostenibilidad medioambiental por parte de diversas universidades (Alba et alii, 2012) la Asamblea de la Conferencia de Rectores de las Universidades Españolas (CRUE) aprobó por unanimidad en 2002 la creación del "Grupo de Trabajo de la CRUE sobre la Calidad Ambiental y el Desarrollo Sostenible” (CRUE, Valladolid, 2005) cuyo principal propósito fue la sensibilización ambiental en las universidades (Geli, 2002).

Esta sensibilización consiste en involucrar a la comunidad universitaria y plantear estrategias y campañas ambientales a todos los niveles de la institución con escasos resultados hasta la fecha (Alfie, 2003; Dagiliūtè, 2018).

Una de las principales estrategias ha sido la introducción de la enseñanza medioambiental como competencias transversales en la Universidad.

\subsection{La introducción de la sostenibilidad entre las competencias transversales influye positivamente en el aumento de la conciencia medioambiental entre los alumnos}

El de 29 octubre (BOE, 2007) por el que se establece la ordenación de las enseñanzas universitarias oficiales en su preámbulo menciona que "los planes de estudios conducentes a la obtención de un título deberán, por tanto, tener en el centro de sus objetivos la adquisición de competencias por parte de los estudiantes".

Desde las perspectivas de los empresarios, las competencias son entendidas como el eje de la política de selección entre los nuevos aspirantes (Delors, 1996). De este modo, la calificación se combina con la capacitación mediante competencias aprehendidas. Las empresas demandan así no solo conocimientos técnicosprofesionales, sino también competencias que llevan a saber hacer y ser (Zarifian, 1999).

De esta forma, el actual sistema de competencias transversales permitiría introducir la sostenibilidad en cada una de las asignaturas de los planes de estudio mediante la aplicación de metodologías, técnicas y recursos pertinentes (Aznar et 
alii, 2014; Martínez et alii, 2007). Así, el Real Decreto 1393/2007, seguidamente se afirma que "se debe tener en cuenta que la formación en cualquier actividad profesional debe contribuir a la protección medioambiental" ya sea a nivel individual o como empresa (Robina-Ramírez, et alii, 2019).

Esta regulación gubernamental permite introducir la cultura de la sostenibilidad en las aulas y en la investigación. De este modo, la enseñanza de la sostenibilidad pasaría a ser uno de los principales desafíos y retos a promover en las universidades (Cebrián et alii, 2014; Hidalgo et alii, 2012). Al fin y al cabo, la universidad desempeña un papel fundamental en la labor educativa y en la trasmisión de valores (Ferrer, 2004; Robina Ramírez y Suárez, 2019) y actualmente, dada la amenaza de agotamiento de recursos naturales, la sostenibilidad se ha convertido en un valor en alza a proteger (Alfie, 2003).

Las competencias educativas en el área medio ambiental realizan una doble función entre los estudiantes. De un lado, permite adquirir unas actitudes que relacionen la naturaleza con la sociedad, y de otro proporciona una capacidad holística de la complejidad que supone relacionar el mundo del trabajo y el mundo natural, la ética pública y la ética personal, la producción y respeto al medio ambiente (Mora Penagos, 2011).

Para relacionar el medioambiente con la dimensión social, laboral y empresarial algunos estudios han identificado diferentes perspectivas de competencias medio ambientales en diseños curriculares y planes de estudios. Entre ellas la resolución de problemas medioambientales, la visión científica del medio ambiente, y la relacional medio ambiente-ciudadanía (Vicent y Focht, 2009). A tenor de lo expresado se plantea testar las dos siguientes hipótesis:

$\mathrm{H}_{1}$. La introducción de competencias medioambientales transversales influye positivamente en el aumento de la conciencia medioambiental entre los alumnos de la universidad.

$\mathrm{H}_{2}$. La introducción de competencias medioambientales transversales influye positivamente en la formación del profesorado de la universidad.

\subsection{La formación de profesores en desarrollo sostenible influye positivamente en el aumento de la conciencia medioambiental entre los alumnos de la universidad.}

En el proceso de involucrar a la comunidad universitaria el profesorado juega un papel esencial como responsables de la educación de los alumnos en las universidades (Alba, 2007). Sin unos profesores bien formados medioambientalmente sería complicado sensibilizar a los alumnos en una conciencia de respeto a la naturaleza (Holmberg and Samuelsson, 2006). 
El reto es, por tanto, integrar la sostenibilidad no solo en la enseñanza regular de los alumnos e investigación, sino también en las operaciones propias a realizar en los campus universitarios, es decir entre los gestores de la propia universidad (Lozano, 2013; Dagiliūtè et alii, 2018).

Para ello es necesario aportar una visión estratégica en materia de educación para el desarrollo sostenible (Cebrián et alii, 2014), tanto en la conexión de la sostenibilidad con las diferentes materias a impartir como en el plan de formación que reciben los profesores (Vilches y Gil, 2007).

Este plan estratégico requiere proporcionar los recursos financieros necesarios para integrar la educación sobre la sostenibilidad en los planes docentes y de investigación (Cebrián et alii, 2014) en un contexto de respeto de su libertad académica para enseñar e investigar en temas relacionados con la sostenibilidad (Cebrián et alii, 2014).

Este proceso de liderazgo en la universidad debe estar acompañado también por la asignación adecuada de responsabilidades y recompensas entre los profesores (Vilches y Gil, 2012). El sistema de incentivos permite a los docentes responder a las necesidades cambiantes de la sociedad (Lozano, 2006). A modo de ejemplo, Aznar et alii (2014) propone ítems pertinentes en los cuestionarios de evaluación de la docencia, como sistemas gratificantes que reconozcan y recompensen las buenas prácticas en sostenibilidad (Aznar, 2014).

Estos sistemas de incentivos permitirían adaptar las tradicionales metodologías de docencias muy distanciadas de la cultura sostenible (Vilches y Gil, 2012) ausentes durante décadas en los procesos de formación de muchos docentes (Macarrón, 2012). Esta estrecha relación entre la formación del profesorado y el desarrollo de la conciencia medioambiental entre los estudiantes de la universidad nos lleva a plantear la siguiente hipótesis.

$\mathrm{H}_{3}$. La formación medioambiental del profesorado influye positivamente en el aumento de la conciencia medioambiental entre los alumnos de la universidad.

\subsection{La incorporación de actividades paralelas sobre concienciación medio ambiental influirá positivamente en el aumento de la conciencia medioambiental entre los estudiantes}

Parte de la misión de la universidad es formar individuos conscientes, responsables y con una actitud positiva de cambio frente al medio ambiente (Alfie, 2003). Motivo por el cual comprender cómo valoran los estudiantes las políticas y enseñanzas medioambientales sirve como indicador para medir la participación y 
actitud de los estudiantes en las posibles iniciativas de sostenibilidad (Nejati y Nejati, 2013).

Hasta la fecha encontramos diferentes estudios que han explorado las percepciones de los estudiantes sobre las medidas de sostenibilidad emprendidas en la Universidad (Nejati y Nejati, 2013; Larrán et alii, 2018; Alonso-Almeida et alii, 2015).

Entre esos estudios Alonso-Almeida et alii (2015) afirma que los alumnos universitarios españoles tienen una alta percepción sobre temas de sostenibilidad. Esta alta percepción medioambiental contrasta con una escasa formación sobre la defensa de recursos naturales y protección del medio ambiente impartida en las universidades (Dagiliūtè et alii, 2018; Martínez, 2008). Es por ello por lo que Martínez (2008) destaca el elevado gap existente entre la alta percepción sostenible de los alumnos y su baja participación en iniciativas medioambientales y sostenibles.

Para ello es interesante conocer cuáles son los factores relevantes que permitirán aumentar el índice de participación en actividades medio ambientales de los estudiantes con el objetivo de introducir las mejoras necesarias en el sistema educativo (Seto-Pamies, 2016). A partir de ahí será necesario formular y adaptar planes de estudios adecuados para influir de manera correcta en los estudiantes y en sus actuaciones en materia de sostenibilidad (Dagiliūtè et alii, 2018).

Algunos ejemplos pueden ser campañas de concienciación, ciclos de conferencias, cine, teatro, exposiciones, actividades promovidas por los equipos de dirección de los centros o mejor aún, por las asociaciones de estudiantes concienciados de la importancia del respeto hacia el medio ambiente en cada una de las actividades que desarrollan (Solís, 2008).

Entre las iniciativas que los alumnos sugieren para aumentar la conciencia medioambiental y participación podemos citar algunas como invitar a expertos y líderes en sostenibilidad para explicar la importancia del respeto al medio, así como la incorporación de casos prácticos en las diversas asignaturas (Pamies, 2016).

Especialmente importante es el desarrollo de la conciencia medioambiental en las áreas de empresa y finanzas de la Universidad (Robina Ramírez y PalosSánchez, 2018b). La futura actividad empresarial en la mayoría de los casos está abocada a un impacto negativo en el medio ambiente. En estos casos, la toma decisiones en las empresas debe ir conectada al respeto al medio ambiente (Matten y Moon, 2004). En este sentido, como señalan Jiménez y Aldeanueva (2016), las empresas que incorporan la sostenibilidad al diseñar sus estrategias son valoradas positivamente, por la sociedad, ya que, teóricamente, se trata de organizacio- 
nes comprometidas con una administración más justa y eficiente de los recursos que ofrece el planeta.

De ahí la importancia de que los futuros gerentes aprendan a gestionar estos impactos (Jallow 2010; Robina Ramírez y Palos-Sánchez, 2018a; Cornelius et alii, 2007; Waples et alii, 2009; Kerr y Hart-Steffes, 2012). Después de todo se espera que las universidades se comprometan con las comunidades y beneficien a la sociedad en general (Lukman y Glavic, 2007).

Para evaluar al papel que juegan estas actividades de concienciación medioambiental en el ámbito universitario se pueden formular las siguientes hipótesis:

$\mathrm{H}_{4}$. La introducción de competencias medioambientales transversales influye positivamente en la organización de actividades universitarias de concienciación medioambiental.

$\mathrm{H}_{5}$. La organización de actividades de respeto a la naturaleza influye positivamente en el aumento de la conciencia medioambiental entre los alumnos de la universidad.

\section{Metodología}

\subsection{Definición de variables}

La tercera sección destaca la metodología de investigación para contextualizar los objetivos del trabajo mediante la definición de las variables, la muestra, las hipótesis del estudio y la propuesta del modelo de estudio.

De acuerdo con la revisión de la literatura del trabajo se eligieron por un grupo de profesores y alumnos diversos constructos para, a partir de ahí, poder definir diversas políticas de sostenibilidad en la Universidad.

Para ello dos "focus groups" fueron organizados para presentar los constructos, así como definir los indicadores. A partir de ahí se fijó en el segundo focus group una metodología de validación de cada uno de los indicadores.

En la primera reunión un grupo de 8 profesores y 12 alumnos debatieron son los indicadores o ítems y los constructos presentados, seleccionados estos a partir de la revisión de la literatura. Los cuatro constructos fueron aprobados. Sin embargo, los indicadores fueron modificados. Dos ítems fueron eliminados y otros dos corregidos para hacerlos más inteligibles a los alumnos.

Los constructos seleccionados fueron cuatro: 1. Desarrollo de la conciencia medioambiental entre estudiantes (DCM). 2. Formación medioambiental del profesorado (FPS). 3. Competencia medioambiental transversal (CMT). 4. Actividades de concienciación medio ambiental (ACM).

Los indicadores relativos a cada uno de los constructos fueron: 
1. Desarrollo de la conciencia medioambiental entre estudiantes (DCM): (DCM1) Incidencia de las políticas de sostenibilidad en la conciencia medioambiental de los estudiantes (Cicmil, Ecclestone y Collins, 2007; People and Work Unit, 2007), (DCM2) incidencia de las competencias medioambientales en la conciencia medioambiental de los estudiantes (Cincera y Krajhanzl, 2013; Aznar et al, 2014; AACSB International, 2015), (DCM3) la introducción de asignaturas sobre medioambiente incide en la conciencia medioambiental de los estudiantes (De la Cuesta, 2004; Colín, 2003; Hart, 2009; Landrum y Edwards, 2011).

2. Formación medioambiental del profesorado (FPS). (FPS1) Formación de docentes deberían estar formados en temas de sostenibilidad (Alba, 2007), (FPS2) Concienciación medioambiental a través de las asignaturas impartidas (Holmberg and Samuelsson, 2006), (FPS3) Integridad del profesorado en torno a su conducta medioambiental (Lozano, 2013), (FPS4) Incentivación del respeto del medioambiente entre los alumnos (Dagiliūtè et alii, 2018; Aznar, 2014).

3. Competencia medioambiental transversal (CMT). (CTM1) Introducción una cultura de respeto hacia la naturaleza de manera transversal en la Universidad (Cebrián et alii 2014; Alfie, 2003), (CTM2) Incidencia de la competencia medioambiental transversal en la futura gestión de empresas (Alfie, 2003; Mora Penagos, 2011), (CTM3) Complemento de los conocimientos teorico-prácticas con las nociones aprendidas en las competencias medioambientales transversales (Vincent y Focht, 2009)

4. Actividades de concienciación medio ambiental (ACM). (ACM1) Análisis de casos prácticos como mecanismo para aumentar el compromiso con el medioambiente (Seto-Pamies, 2016; Dagiliūtè et alii, 2018), (ACM2) La incorporación de ciclos de conferencias, cursos, exposiciones contribuye a concienciar medioambientalmente a los estudiantes (Solís, 2008; Pamies, 2016; Matten \& Moon, 2004), (ACM3) Actividades de concienciación en la universidad para tomar decisiones responsables sobre el uso de los recursos naturales (Jiménez y Aldeanueva, 2016.)

Con el objetivo era validar cada uno de los ítems así como recoger la opinión de profesores y alumnos en el segundo "focus group" se convocó a un mayor grupo de profesores y alumnos procedente de las diferentes áreas de conocimiento. En total fueron 22 profesores y 64 alumnos. Todos los ítems fueron aprobados por más del $80 \%$ de los participantes, superando así la cifra de $70 \%$ inicialmente establecida de validación. En la tabla 1 se muestran los porcentajes obtenidos finalmente. 
Tabla 1. Validación de los ítems.

\begin{tabular}{lcc}
\hline Items & Profesores & Alumnos \\
\hline DCM1 & $90 \%$ & $81 \%$ \\
DCM2 & $91 \%$ & $83 \%$ \\
DCM3 & $95 \%$ & $82 \%$ \\
FPS1 & $90 \%$ & $94 \%$ \\
FPS2 & $91 \%$ & $93 \%$ \\
FPS3 & $87 \%$ & $92 \%$ \\
CTM1 & $94 \%$ & $99 \%$ \\
CTM2 & $93 \%$ & $95 \%$ \\
CTM3 & $90 \%$ & $94 \%$ \\
ACM1 & $85 \%$ & $91 \%$ \\
ACM2 & $86 \%$ & $90 \%$ \\
ACM3 & $84 \%$ & $87 \%$ \\
\hline
\end{tabular}

Los ítems se transformaron en preguntas medidas través de la escala de Likert de diez puntos utilizada para indicar el grado de importancia de los factores, desde 1 ("totalmente en desacuerdo") a 10 ("muy de acuerdo") (Allen, 2007).

\subsection{Población y muestra}

La población a la que nos dirigimos fue de 1800 estudiantes en la Facultades de Económicas y 1450 en la Facultad de Empresa, Finanzas y Turismo. Para seleccionar el modo de llegar a todos los alumnos se dirigieron dos cartas a los decanos de ambas facultades explicándole el objetivo de la investigación. A partir de ahí se procedió a enviar un correo electrónico a todos los profesores de cada una de las áreas de conocimientos pidiéndoles su colaboración para acceder a sus clases y explicar directamente el objetivo de la investigación. Un 60\% de los profesores dela Facultad de Económicas de Badajoz accedió colaborar con el desarrollo de la investigación en sus clases. En el caso de la Facultad de Empresa, Finanzas y Turismo el porcentaje subió al $85 \%$.

Durante la realización de la actividad de investigación los alumnos tenían plena libertad para abandonar la clase. La muestra obtenida fue de 455 estudiantes. Finalmente, 35 cuestionarios no fueron correctamente rellenados por lo que tuvieron que ser eliminados. La muestra definitiva fue de 420 alumnos (ver tabla 2).

Un 55\% de los alumnos participantes fueron mujeres. La mayoría de los alumnos (91\%) tenían menos de 22 años. El mayor porcentaje de ellos está estu- 
diando Administración de la Empresa (35\%). Entre el total de participantes un $60 \%$ estudia primer y segundo curso.

Los cuestionarios fueron recogidos en dos campus distintos de la Universidad de Extremadura. La participación de los alumnos en la investigación se desarrolló a lo largo del mes febrero y marzo de 2019. Se seleccionó este período de muestreo porque coincide con el comienzo del segundo semestre. Ello facilitó que los alumnos puedan participar sin estar sujetos a las presiones de los exámenes.

Tabla 2. Variables relacionadas con la adscripción de los estudiantes en la Universidad

\begin{tabular}{|c|c|c|}
\hline Información & $\mathrm{N}=420$ & Porcentaje (\%) \\
\hline \multicolumn{3}{|l|}{ Genero } \\
\hline Masculino & 191 & $45 \%$ \\
\hline Femenino & 229 & $55 \%$ \\
\hline Total & 420 & $100 \%$ \\
\hline \multicolumn{3}{|l|}{ Edad } \\
\hline 18 años o inferior & 139 & $33 \%$ \\
\hline 19-20 años & 107 & $25 \%$ \\
\hline 21-22 años & 96 & $23 \%$ \\
\hline Más de 23 & 78 & $19 \%$ \\
\hline Total & 420 & $100 \%$ \\
\hline \multicolumn{3}{|l|}{ Titulación } \\
\hline Administración de Empresas & 149 & $35 \%$ \\
\hline Económicas & 124 & $30 \%$ \\
\hline Finanzas y Contabilidad & 85 & $20 \%$ \\
\hline Turismo & 62 & $20 \%$ \\
\hline Total & 420 & $100 \%$ \\
\hline \multicolumn{3}{|l|}{ Curso } \\
\hline $1^{\circ}$ & 142 & $34 \%$ \\
\hline $2^{\circ}$ & 108 & $26 \%$ \\
\hline $3^{\circ}$ & 92 & $22 \%$ \\
\hline $4^{\circ}$ & 72 & $17 \%$ \\
\hline Master & 6 & $1 \%$ \\
\hline Total & 420 & $100 \%$ \\
\hline
\end{tabular}




\subsection{Hipótesis y modelo}

Las hipótesis recogidas en la revisión de la literatura se exponen a continuación. Estas se pueden visualizan en el modelo estructural de constructos expresado en la Figura 1.

$\mathrm{H}_{1^{+}}$La introducción de competencias medioambientales transversales influye positivamente en el aumento de la conciencia medioambiental entre los alumnos de la universidad.

$\mathrm{H}_{2^{*}}$ La introducción de competencias medioambientales transversales influye positivamente en la formación del profesorado de la universidad.

$\mathrm{H}_{3}$. La formación medioambiental del profesorado influye positivamente en el aumento de la conciencia medioambiental entre los alumnos de la universidad.

$\mathrm{H}_{4^{*}}$ La introducción de competencias medioambientales transversales influye positivamente en la organización de actividades universitarias de concienciación medioambiental.

$\mathrm{H}_{5^{*}}$ La organización de actividades de respeto a la naturaleza influye positivamente en el aumento de la conciencia medioambiental entre los alumnos de la universidad.

Figura 1. Modelo estructural.

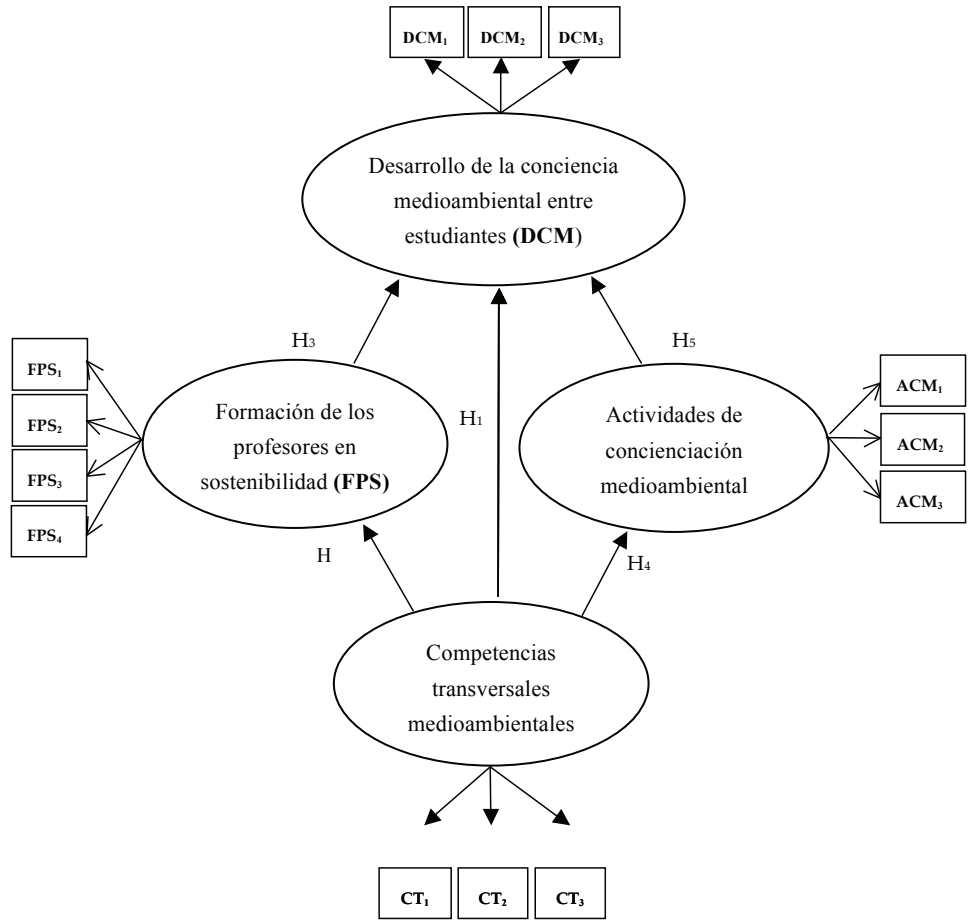


Los datos obtenidos a través de los cuestionarios se analizaron utilizando la herramienta informática SmartPLS 3 la cual es especialmente recomendada para modelos compuestos o construcciones (Rigdon, Sarstedt, Ringle, 2017). Esta técnica estadística se aplica cuando los datos se estructuran en una serie de relaciones de dependencia interrelacionadas entre las variables latentes y los indicadores (Sarstedt, Hair, Ringle, Thiele, Gudergan, 2016).

\section{Análisis de los resultados}

\subsection{Resultados del modelo de medida}

El enfoque PLS está definido por dos modelos: el modelo de medición y el modelo estructural. Como paso preliminar al análisis del modelo estructural, es necesario analizar la confiabilidad y validez del modelo de medición (Hair et alii, 2016). El procedimiento para la evaluación de los modelos de medición de los elementos reflectantes se muestra en la Tabla 5. La confiabilidad se evaluó mediante el examen de cargas individuales o correlaciones simples de las medidas con sus respectivas variables latentes (se aceptó $\geq 0.7$ ). El coeficiente alfa de Cronbach se usó como un índice de confiabilidad de las variables latentes.

Además, se calculó la fiabilidad compuesta. La validez convergente de las variables latentes se evaluó a través de la inspección de la varianza promedio extraída (AVE) (aceptado> 0.5). La validez discriminante de las variables latentes se verificó utilizando el criterio de Fornell-Larcker (Fornell y Bookstein, 1982) al examinar si la raíz cuadrada del valor promedio extraído (AVE) de cada ítem estaba por encima de las correlaciones con las otras variables latentes.

La Tabla 3 también muestra que la raíz cuadrada de la varianza promedio extraída (AVE) para cada construcción es mayor que su correlación más alta con cualquier otra construcción.

Tabla 3. Confiabilidad, validez de los constructos.

\begin{tabular}{|c|c|c|c|c|c|c|c|c|}
\hline \multirow[t]{2}{*}{ Variables } & \multirow[t]{2}{*}{ Alfa de Cronbach } & \multirow[t]{2}{*}{ rho_A } & \multirow[t]{2}{*}{$\mathrm{CR}$} & \multirow[t]{2}{*}{ AVE } & \multicolumn{4}{|c|}{ Fornell-Larcker Criterion } \\
\hline & & & & & DCM & CT & $\mathrm{ACM}$ & FPS \\
\hline DCM & 0.855 & 0.862 & 0.856 & 0.665 & 0.816 & & & \\
\hline $\mathrm{CT}$ & 0.834 & 0.835 & 0.835 & 0.627 & 0.535 & 0.792 & & \\
\hline $\mathrm{ACM}$ & 0.852 & 0.855 & 0.852 & 0.657 & 0.757 & 0.702 & 0.811 & \\
\hline FPS & 0.853 & 0.854 & 0.851 & 0.589 & 0.654 & 0.486 & 0.753 & 0.767 \\
\hline
\end{tabular}


Además, según Henseler, Ringle y Sarstedt (2015), se realizó una prueba para demostrar que la falta de validez discriminante se detecta mejor con otra técnica. Esta prueba se llama relación heterotrait-monotrait (HTMT). La Tabla 4 muestra que las relaciones HTMT para cada par de factores fueron $<0.90$ (Henseler, 2017).

\begin{tabular}{|c|c|c|c|c|}
\hline \multicolumn{5}{|c|}{ Heterotrait-Monotrait Ratio (HTMT) } \\
\hline & $\mathrm{DCM}$ & CT & $\mathrm{ACM}$ & FPS \\
\hline \multicolumn{5}{|l|}{ DCM } \\
\hline CT & 0.536 & & & \\
\hline $\mathrm{ACM}$ & 0.755 & 0.701 & & \\
\hline FPS & 0.652 & 0.482 & 0.749 & \\
\hline
\end{tabular}

\subsection{Resultados del modelo estructural}

Después de examinar el modelo de medición, se analizaron las relaciones entre las construcciones. Se estudiaron los coeficientes de trayectoria de las hipótesis. Se realizó un programa de inicio de 5000 submuestras para verificar la significación estadística de cada ruta. La varianza explicada $\left(R^{2}\right)$ de las variables latentes endógenas y el valor $\mathrm{p}$ de los coeficientes de regresión (prueba t) se utilizaron como indicadores del poder explicativo del modelo (Tabla 5). Los resultados obtenidos nos permitieron aceptar todas las hipótesis, ya que no hubo diferencias estadísticamente significativas en las relaciones entre las variables en nuestro modelo (valor de $\mathrm{p}>0.05$ ).

Tabla 5: Path coefficients

\begin{tabular}{cccccc}
\hline Hypotheses & $\beta$ & Lower CI & Higher CI & t Statistic & p-value \\
\hline $\mathrm{H}_{1}$ CMT $\rightarrow$ DCM & 0.346 & 0.181 & 0.506 & 4.133 & 0.000 \\
$\mathrm{H}_{2}$ CMT $\rightarrow$ FPS & 0.486 & 0.342 & 0.611 & 6.926 & 0.000 \\
$\mathrm{H}_{3}$ FPS $\rightarrow$ DCM & 0.368 & 0.168 & 0.543 & 3.977 & 0.000 \\
$\mathrm{H}_{4}$ CMT $\rightarrow$ ACM & 0.535 & 0.398 & 0.663 & 7.721 & 0.000 \\
$\mathrm{H}_{5}$ ACM $\rightarrow$ DCM & 0.332 & 0.188 & 0.471 & 4.643 & 0.000 \\
\hline
\end{tabular}

Statistical significance: ${ }^{*} \mathrm{p}<0.05 ;{ }^{* *} \mathrm{p}<0.01 ;{ }^{* * *} \mathrm{p}<0.001$; n.s: not significant.

The following figures (Figure 2) show the result for the model and the hypotheses that were analyzed in the model. 
Figura 2. Resultados del algoritmo PLS

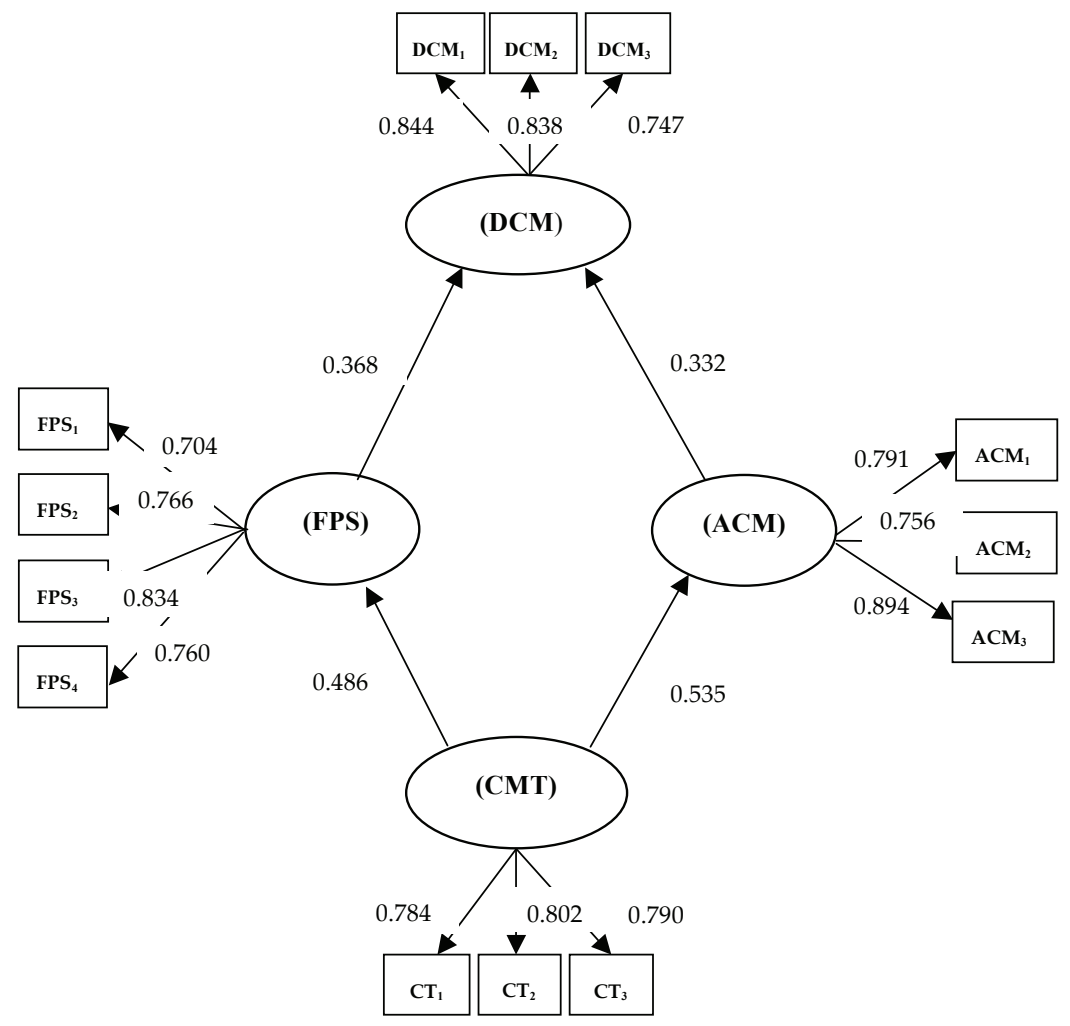

Nota: DCM: Desarrollo de la conciencia medioambiental entre estudiantes. CMT: Competencias medioambientales transversales. FPS: Formación de los profesores.

ACM. Actividades de concienciación medioambiental.

\subsection{Test de bondad de ajuste para el modelo}

Primero, se evaluó el ajuste general del modelo utilizando el indicador residual medio de la raíz cuadrada estándar (SRMR). Hu y Bentler (1998) definen la SRMR como la discrepancia media cuadrática media entre las correlaciones observadas y las correlaciones implícitas en el modelo. SRMR se puede utilizar para evaluar el ajuste general de un modelo de investigación en PLS y evitar una especificación incorrecta del modelo (Henseler, Hubona, Ray, 2016). Un valor de corte de 0.08 para SRMR se considera el más apropiado en PLS (Henseler, Hubona, Ray, 2016). En este estudio, el SRMR fue de 0.068, lo que significa que el modelo se ajusta a los datos empíricos (Hair et alii, 2016). 
Los valores de $\mathrm{R}^{2}$ (ver Tabla 6) obtenidos para la investigación llevaron a las siguientes conclusiones: 0.67 “Sustancial”, 0.33 “Moderado" y 0.19“Débil” (Chin, 1988). El resultado obtenido para la variable principal dependiente en el modelo de intención de uso (DCM) fue $\mathrm{R}^{2}=77.1 \%$. Por lo tanto, la evidencia muestra que el modelo presentado tiene una capacidad predictiva sólida o sustancial. Esto explica por qué tanto las actividades de concienciación medioambiental (ACM) como la formación del profesorado en temas medioambientales (FPS) son dos factores que contribuyen en un mejor desarrollo de la conciencia medioambiental entre los estudiantes (DCM).

Tabla 6. Coeficiente de determinación $\left(\mathrm{R}^{2}\right)$ y prueba de Stone-Geisser $\left(\mathrm{Q}^{2}\right)$

\begin{tabular}{ccc}
\hline Constructo & $\mathrm{Q}^{2}$ & $\mathrm{R}^{2}$ \\
\hline DCM & 0.435 & 0.771 \\
CT & & \\
ACM & 0.146 & 0.287 \\
FPS & 0.103 & 0.236 \\
\hline
\end{tabular}

La técnica de Blindfolding consiste en omitir parte de los datos de un constructo dado durante la estimación de los parámetros y luego tratar de estimar lo que se omitió de los parámetros estimados (Chin, 1998). De esta manera, la relevancia predictiva del modelo se estudió mediante el uso del, se demostró que el modelo tiene capacidad predictiva. Como puede verse en la Tabla 6, todas las construcciones endógenas cumplen con $\mathrm{Q}^{2}>0$. Siguiendo la prueba de StoneGeisser $\left(\mathrm{Q}^{2}\right)$ (Stone, 1974; Geisser, 1974; Hair et alii 2016.) establecen valores de 0.02 como valores pequeños, valores de 0.15 como valores medios y valores 0.35 como valores grandes para considerar validez predictiva del modelo) los valores se fijan en tres pasos; $0.02,0.15$ y 0.35 . Indica relevancia predictiva pequeña, media y alta. Como resultado, todas las construcciones tienen relevancia predictiva, ya que los valores de $\mathrm{Q}^{2}$ son todos mayores que 0.02 .

\section{Discusión}

Debido al escaso el número de universidades que han implementado enseñanzas para un comportamiento medioambiental responsable en España (Lozano et alii, 2013) nuevas experiencias educativas se han planteado para desarrollar la conciencia medioambiental en la universidad (Boeve-de Pauw y Van Petegem, 2011, Olsson et alii, 2016; People and Work Unit, 2007; Robina-Ramírez y MedinaMerodio, 2019). 
En este sentido, el estudio propone un modelo significativo que permita abrir nuevas vías de enseñanza de sostenibilidad en la Universidad y mejorar las actitudes medioambientales de los estudiantes. Para ello se han testado cuatro variables latentes entre la comunidad de estudiantes en las Facultades de Economía y de Empresa, Finanzas.

A partir de los datos obtenidos, el modelo estructural ha resultado muy significativo con un coeficiente de determinación, $\mathrm{R}^{2}=77.1 \%$. $\left(\mathrm{R}^{2}>0,67\right)$ (Chin, 1988).

$\mathrm{Al}$ mismo tiempo, el modelo expresa una alta capacidad predictiva alta $\left(\mathrm{Q}^{2}=\right.$ $0.435)$. El modelo genera predicciones precisas sobre los potenciales resultados en nuevas observaciones (Shmueli y Koppious, 2011), lo cual es interesante para replicar el estudio en otras Facultades.

Todas las hipótesis del modelo ( $\mathrm{H} 1$ a H5) son significativas y, por lo tanto, son aceptadas. Esto significa en primer lugar que el desarrollo de competencias medioambientales transversales en las áreas de economía y empresa influyen positivamente en desarrollo de la conciencia medioambiental (H1: CMT $\rightarrow \mathrm{DCM}$; $\beta=0.34 ; t=4.133)$. Estos datos corroboran el contenido de la valoración realizada por alumnos y profesores en el proceso de validación de la encuesta. Alumnos expresaron que "la adquisición de competencias es lo que realmente va a ser valorado en un futuro por la empresa", otros argumentaron que "los conocimientos sin competencias profesionales y medioambientales adquiridas no se traducirán en la consecución de puestos de trabajo". Otro alumno afirmó que "solo los conocimientos sobre la necesidad de proteger el medioambiente no son suficientes, es necesario aprender a desarrollar actos concretos de defensa y respeto hacia el medio ambiente". Estos ejemplos recogidos en las entrevistas cualitativas con los alumnos durante el segundo focus group nos aportan una perspectiva muy interesante para enseñar sostenibilidad a través de metodologías y técnicas transversales en la universidad (Aznar et alii, 2014; Martínez et alii, 2007).

No solo implicaciones teóricas de extraen de estas conclusiones, prácticas también. Así, estas competencias medioambientales podrían ser incluidas en diseños curriculares y planes de estudios. Entre ellas figuran la resolución de problemas medioambientales, la visión científica del medio ambiente, y la relacional medio ambiente-ciudadanía (Vincent y Focht, 2009). Para ello es necesario llegar a una conexión de la sostenibilidad con las diferentes materias a impartir como en el plan de formación que reciben los profesores (Vilches y Gil, 2007) como parte de una visión estratégica en materia de educación para el desarrollo sostenible (Cebrián et alii, 2014). 
En segundo lugar, los resultados muestran como la incorporación de competencias medioambientales transversales en la Universidad influiría positivamente en la mejor formación de los profesores en responsabilidad medioambiental $(\mathrm{H} 2$ : CMT $\rightarrow$ FPS; $\beta=0.486 ; t=6.926)$, $y$ en la puesta de marcha de actividades de concienciación medioambiental (H4: CMT $\rightarrow \mathrm{ACM} ; \beta=0.535 ; \mathrm{t}=7.721$ ).

En relación a la incidencia de competencias medioambientales transversales (CMT) en la formación de los profesores (FPS), las entrevistas realizadas fueron muy clarificadoras. En la línea de los expresado por Holmberg and Samuelsson, (2006), en relación a la necesaria formación medioambiental del profesorado, los alumnos destacan por un lado el "escaso interés del profesorado por sensibilizar a los alumnos en sostenibilidad" y por otro, "la ausencia de políticas en la propia universidad que ayuden a los alumnos a entender la importancia de gestionar sosteniblemente los recursos". Aspectos ampliamente debatidos por Lozano, (2013) y Dagiliūtè et alii, (2018) en la misma línea argumental.

Por otro lado, en relación con la incidencia de competencias medioambientales transversales (CMT) en la práctica de actividades de concienciación medioambiental (ACM), se puede afirmar que la alta percepción que tienen los universitarios en temas de sostenibilidad (Alonso-Almeida et alii, 2015) no se corresponde con una escasa oferta de actividades medioambientales. En concreto los alumnos se refieren a actividades de limpieza de ríos, ciclo de conferencias, etc. Es patente que, como señala un estudiante, "la falta de claridad de ideas y liderazgo por parte del profesorado para incorporar actividades de concienciación y respeto de la naturaleza”. Este giro hacia el respeto medioambiental debería comenzar por la asunción de responsabilidades y tareas estructuradas desde la dirección de la Universidad (Vilches y Gil, 2012).

\section{Conclusión}

La primera conclusión a la que llegamos con este trabajo de investigación es el escaso eco que hasta la fecha ha tenido la competencia sobre la protección medioambiental desarrollada en el Real Decreto 1393/2007. En concreto en las facultades de economía y empresa.

En segundo lugar, y como consecuencia de la primera conclusión, la falta de recursos económicos y académicos concretados en efectivas políticas de sostenibilidad en la Universidad. A juicio de los alumnos no solo escasean las referencias medioambientales en la enseñanza transversal de las asignaturas las áreas de economía y empresa sino también es llamativa la ausencia de políticas de sostenibilidad en la Universidad conocidas por los alumnos (Nejati y Nejati, 2013; UN, 2012; Beland Lindahl, 2016; Ull, et alii, 2010). Para ello sería necesario es- 
tablecer políticas y programas formativos transversales para insertar contenidos de defensa del medio ambiente en aquellas asignaturas más relacionadas con la gestión de empresa y gestión de recursos naturales.

El tercer objetivo está relacionado directamente con la filosofía empresarial impartida actualmente en las facultades de economía y empresa. Los alumnos afirman que "la maximización del beneficio y la reducción de costes son los únicos criterios utilizados para la toma de decisiones empresariales". Junto a ello, otros alumnos añaden que "si la protección del medioambiente genera un mayor coste, prevalece el criterio económico sobre el medioambiental". Aspectos ambos que señalan la gran distancia existente entre la maximización económica y el respeto del medio ambiente (Matten y Moon, 2004). Estas afirmaciones son un botón de muestra de la gran responsabilidad que la universidad tiene actualmente de formar estudiantes que actúen de forma consecuente con el medio ambiente (Cornelius et alii, 2007; Waples et alii, 2009).

Diversas limitaciones en la realización del estudio deben ser señaladas. En primer lugar, la reacción negativa de parte del profesorado para recibir formación que les permitan incorporar ejemplos de conductas medioambientalmente responsables en las asignaturas que actualmente imparten. De hecho, casi un $40 \%$ de los profesores preguntados no se mostraron abiertos para recibir una formación medioambiental que les permita incorporar la perspectiva medioambiental en sus clases. En segundo lugar, encontramos la misma falta de interés en los directivos de la Universidad para recibir formación oportuna que les permita desarrollar estrategias de sostenibilidad que permita hacer un uso más racional de los recursos.

A modo de conclusión, esta investigación ha revelado como los alumnos son proclives a recibir nociones y organizar actividades que les permitan desarrollar un modelo de desarrollo más habitable en consonancia con entornos medioambientales responsables. Sin embargo, a nivel educativo aún estamos lejos de este objetivo. Las principales barreras se erigen desde el profesorado y la dirección de la universidad. Sin embargo, el cambio de mentalidad ya existe entre los jóvenes alumnos, aunque actualmente está lastrado por una generación de docentes que aún se rigen por modelos de enseñanza más en criterios economicistas. El reto está en transformar a los educadores de la universidad a partir de modelos de enseñanza medioambiental entre los futuros dirigentes de empresas en nuestro país. 


\section{Referencias}

Alba, David, Barbeitos, Rosa, Barral, Teresa, Benayas, Javier, Blanco, Dora, Domènech, Xavier, Fernández, Isabel, Florensa, Anna, García, Fuensanta, López, Noelia and Ysern, Pere (2012). "Strategies for sustainability and social responsibility in Spanish universities: a tool for evaluation". Teacher: Journal of Curriculum and Teacher Training, 16(2):59-75.

Alba, David. "Analysis of management and educational processes for sustainability in public Spanish universities". Research trends in Environmental Education Relating to Socio-Educational and Community Development, Autonomous National Parks Organisation, Environment Ministry. 2007. 197-215. [Accesed on 10 February 2019]. Available at: <www.shiftt.com/ redfia/bibliotecavirtual/ministerio\%20de\%20ambiente\%20Espa\%C3\%B1a/ tendencias/tendencias_paginas197_215.pdf $>$.

Alfie, Miriam (2003)."Medio ambiente y universidad: retos y desafíos ambientales en la Universidad Autónoma Metropolitana-Azcapotzalco". El Cotidiano, 19 (122): 86-92.

Allen, Elaine and Seaman, Christopher, (2007). "Likert scales and data analyses". Quality progress, 40(7), 64-65.

Alonso-Almeida, María del Mar, Fernández de Navarrete, Francisco, Rodriguez-Pomeda, Jesús (2015). "Corporate social responsibility perception in business students as future managers: a multifactorial analysis". Business Ethics: A European Review, 24(1): 1-17.

Association to Advance Collegiate Schools of Business (AACSB International) (2015), "Eligibility procedures and accreditation standards for business accreditation". [Accesed on 10 February 2019] Available at: <https://www.aacsb.edu/-/media/aacsb/docs/accreditation/business/ standards-and-tables/2018-business-standards.ashx?la > .

Aubert, Jaques and Gilbert, Patrick (2003). L'evaluation des competences. Sprimont. Belgique: Mardaga Editions.

Aznar, Pilar, Ull, Angels, Piñero, Albert y Martínez, Pilar (2014).“La sostenibilidad en la formación universitaria: Desafíos y oportunidades". Educación XX1, 17 (1): 133-158.

Beland, Karin, Baker, Susan, Rist, Lucy, and Zachrisson, Anna (2016). "Theorising pathways to sustainability". International Journal of Sustainable Development \& World Ecology, 23(5):399-411.

Boeve-de Pauw, Jelle and Van Petegem, Peter (2011). "The Effect of Flemish Eco-schools on Student Environmental Knowledge, Attitudes, and Affect." International Journal of Science Education, 33(11): 1513-1538. 
Boletín Oficial del Estado (B.O.E*) (2007). "Real Decreto 1393/2007, de 29 de octubre, por el que se establece la ordenación de las enseñanzas universitarias oficiales". Boletín Oficial del Estado, 30; 44037-44048.

Calvo, Susana, y Corrales, Jose (1999). El libro blanco de la educación ambiental en España. Madrid: MMA.

Cebrián, G., Grace, M., and Humphris, D. (2015). Academic staff engagement in education for sustainable development. Journal of Cleaner Production, 106, 79-86.

Cebrián, Gisela, Marcus, Grace and Debra Humphris (2015). "Academic staff engagement in education for sustainable development". Journal of Cleaner Production 106:79-86.

Chin, Wynne (1998). "The partial least squares approach to structural equation modelling". Modern methods for business research, 295(2): 295-336.

Cicmil, Svetlana and Hodgson, Damian (2007). "Risks of innovation in management education: Introducing a critical management perspective onto a project management MBA elective". In IRNOP XII conference (international research network on projects): Projects in innovation, innovation in projects. 19th -21st September 2007. [Accessed on 19 February 2019]. Available at: $<$ http://eprints.uwe.ac.uk/16880>.

Cincera, Jan and Jan Krajhanzl (2013). "Eco-Schools» what factors influence pupils' action competence for pro-environmental behavior?" Journal of Cleaner Production, 61: 117-121. [Accessed 11 February 2019]. DOI: 10.1016/j. jclepro.2013.06.030.

Colín, Leticia (2003). "Deterioro ambiental vs desarrollo económico y social". Artículos técnicos, 103: 1-6.

Cornelius-White, Jeffrey (2007). "Learner-centered teacher-student relationships are effective: A meta-analysis". Review of educational research, $77(1): 113-143$.

Dagiliūté, Renata, Genovaité, Liobikiené and Audroné Minelgaitė (2018). "Sustainability at universities: Students' perceptions from Green and Non-Green universities”. Journal of Cleaner Production, 181: 473-482.

De la Cuesta González, Marta (2004). "El porqué de la responsabilidad social corporativa". Boletín económico de ICE, 2813: 45-58.

Delors, Jaques (1996). La Educación Encierra un Tesoro. París: Unesco Editions. Ferrer, Elsi, Lazo, Jesús and Pierra, Allán (2004). "Universidad y Desarrollo Sostenible". Pedagogía Universitaria, 9(3): 86-95. 
Fornell, Caes and Bookstein, Fred (1982). "Two structural equation models: LISREL and PLS applied to consumer exit-voice theory". Journal of Marketing research, 19(4): 440-452.

Geisser, Seymour (1974). "A predictive approach to the random effect model". Biometrika, 61(1), 101-107.

Geli, Ana María (2002). "Universidad, sostenibilidad y ambientalización curricular". En E. Arbat y A. M Geli (eds.), Ambientalización curricular de los estudios superiores. Tomo I. Aspectos Ambientales de las Universidades. Universitat de Girona: Servei de Publiacions/ Red-ACES.

Giddings, Bob, Hopwood, Bill, O’Brien and Geoff (2002). "Environment, economy and society: fitting them together into sustainable development". Sustainable Development, 10 (4): 187-196. [Accessed 10 February 2019]. DOI: $10.1002 /$ sd.199.

Gutiérrez, José, Benayas, Javier y Calvo, Susana (2006). “Educación para el desarrollo sostenible: evaluación de retos y oportunidades del decenio 2005 2014". Revista Iberoamericana de educación, 40(1)ः25-60.

Hair Jr, Joseph; Hult, Thomas; Ringle, Christian and Sarstedt, Marko (2016). A primer on partial least squares structural equation modeling (PLSSEM). Sage Publications.

Hargreaves, Andy and Giles, Corrie (2003). "The knowledge society school: An endangered entity". In Hargreaves, Andy. Teaching in the knowledge society: Education in the age of insecurity. Maidenhead and Philadelphia: Open University Press.

Hart, Stuart (2009). "Converging on green". BizEd, 8(4), 24-28.

Henseler, J.; Ringle, Christian and Sarstedt, Marko (2015)."A new criterion for assessing discriminant validity in variance-based structural equation modeling". Journal of the academy of marketing science, 43(1), 115-135.

Henseler, Jörg; Hubona, Geoffrey and Ray, Pauline (2016). "Using PLS path modeling in new technology research: updated guidelines". Industrial management $E$ data systems, 116(1), 2-20.

Henseler, Jörg (2017). "Bridging design and behavioral research with variancebased structural equation modelling" Journal of advertising, 46(1): 178-192.

Hidalgo, David, Barbeitos, Rosa, Barral, Teresa, Benayas, Javier, Blanco, Dora, Doménech, Xavier and Ysern, Pere (2012). "Estrategias de sostenibilidad y responsabilidad social en las universidades españolas: una herramienta para su evaluación". Profesorado: Revista de Currículum y Formación de Profesorado, 16(2): 59-75. 
Holmberg, John and Samuelsson, Bo (eds.) (2006). Drivers and Barriers for Implementing Sustainable Development in Higher Education: Göteborg Workshop. December 7-9, 2006 [United Nations Decade of Education for Sustainable Development (2005-2114)]. Unesco.

Hu, Li-tze and Bentler, Peter (1998). "Fit indices in covariance structure modeling: Sensitivity to underparameterized model misspecification". Psychological methods, 3(4): 424 .

JALlow, Kumba (2010). "Education for environmentally responsible behaviour in business". International Journal of Environment and Sustainable Development, $9(1-3) \div 213-227$.

Jiménez, Jos y Aldeanueva, Ignacio (2016). Dirección estratégica internacional. Madrid: Pirámide.

Kerr, Kathleen and Hart-Steffes, J. S. (2012). Sustainability, student affairs, and students. New Directions for Student Services, 137:7-17.

Landrum, Nancy and Edwards, Sara (2011). "Is strategic management (still) responsible for the demise of society?" International Journal of Business Insights E Transformation, 3(3): 62-69

Larrán, Manuel, Andrades and Javier and Herrera, Jesús (2018). "An examination of attitudes and perceptions of Spanish business and accounting students toward corporate social responsibility and sustainability themes". Revista de Contabilidad, 21(2):196-205

Lozano, Rodrigo (2006). "Incorporation and institutionalization of sustainable development into universities: breaking through barriers to change". Journal of cleaner Production, 14 (9-11): 787-796.

Lozano, Rodrigo, Lukman, Rebeca, Lozano, Francisco, Huisingh, Donald and Lambrechts, Wim (2013). "Declarations for sustainability in higher education: becoming better leaders, through addressing the university system". Journal of Cleaner Production, 48: 10-19.

Lukman, Rebeca and Glavič, Peter (2007). "What are the key elements of a sustainable university?" Clean Technologies and Environmental Policy, 9 (2): 103-114.

Macarrón, Leonor (2012). "La educación ambiental o la educación para el desarrollo sostenible: su interpretación desde la visión sistémica y holística del concepto de medio ambiente". Educación y futuro: revista de investigación aplicada y experiencias educativas, $26: 17-42$.

Martínez, Antonio (2008). "La conciencia ambiental como herramienta para la educación ambiental: conclusiones y reflexiones de un estudio en el ámbito universitario". Universidad de Córdoba. 
Martínez, Pilar, Aznar, Pilar, Ull, Ángels y Piñero, Albert (2007). "Promoción de la sostenibilidad en los currícula de la enseñanza superior desde el punto de vista del profesorado: un modelo de formación por competencias". Educatio Siglo XXI, 25: 187-208.

Matten, Dirk and Moon, Jeremy (2004)."Corporate social responsibility education in Europe". Journal of Business Ethics, 54(4): 323-337.

Mora Penagos, William (2011). "La inclusión de la dimensión ambiental en la educación superior: un estudio de caso en la Facultad de Medio Ambiente de la Universidad Distrital en Bogotá".

Nejati, Mostafa and Nejati, Mehran (2013). "Assessment of sustainable university factors from the perspective of university students". Journal of Cleaner Production, 48: 101-107.

Olsson, Daniel, Gericke, Niklas and Rundgren, Chang, (2016). "The effect of implementation of education for sustainable development in Swedish compulsory schools assessing pupils' sustainability consciousness". Environmental Education Research, 22(2): 176-202. [Accessed 15 February 2019] DOI 10.1080/13504622.2015.1005057.

Organización de las Naciones Unidas (ONU) (2015). “Objetivos de Desarrollo Sostenible. 17 objetivos para transformar nuestro mundo de la Agenda 2030". [Accessed 15 February 2019] Disponible en: <http://www.un.org/ sustainabledevelopment/es/objetivos-de-desarrollo-sostenible $>$.

Organización de Naciones Unidas (ONU) (2018). "The Sustainable Development Goals Report 2018”. [Accessed 15 February 2019] Disponible en: <https://unstats.un.org/sdgs/files/report/2018/TheSustainableDevelo pmentGoalsReport2018-EN.pdf $>$.

Pamies, Dolors (2016). "La ética y la responsabilidad social en la formación universitaria: ¿cuál es la percepción de los estudiantes?”. [Consultado el 3 febrero 2019] Disponible en: <http://www.eben-spain.org/docs/Papeles/XXI/laetica-y-la-responsabilidad-social-en-la-formacion-universitaria.pdf $>$.

People and Work Unit (2007). "Evaluation of the Eco-schools Programme in Wales". Final Report. August 2007. Abergavenny. Available at: <http:// www.youngreporters.org/Servicepmenu/InternalpPages/Nationalpdocuments/EcoSchoolsEvaluationReportWales.pdf $>$.

RaUch, Franz (2002). "The potential of education for sustainable development for reform in schools". Environmental Education Research, 8 (1), 43-51. [Accessed 15 February 2019] DOI 10.1080/13504620120109646. 
Rigdon, Edward, Sarstedt, Marko and Ringle, Christian (2017). "On comparing results from CB-SEM and PLS-SEM: Five perspectives and five recommendations". Marketing ZFPS, 39(3): 4-16.

Robina-Ramírez, R. (2016). Consumo Ético y Responsable del Agua en la Península de Yucatán, Comisión Nacional del Agua. CONAGUA, Gobierno de la República de México. Secretaría de Medio Ambiente y Recursos Naturales, ISBN: 978-607-626-039-5

Ramírez, R. R., Seeliger, L*, and Di Pietro, F* (2016). "Price, virtues, principles: How to discern what inspires best practices in water management? A case study about small farmers in the Yucatan Peninsula of Mexico." Sustainability, 8(4), 385.

Robina Ramirez, Rafael and Palos-Sanchez, Pedro (2018a). "Willingness to Comply with Corporate Law: An Interdisciplinary Teaching Method in Higher Education". Sustainability, 10(6): 1991. DOI: 10.3390/su10061991.

Robina Ramírez, Rafael and Palos-Sánchez, Pedro(2018b)."Environmental Firms' Better Attitude towards Nature in the Context of Corporate Compliance". Sustainability, 10(9), 3321. DOI: 10.3390/su10093321

Robina-Ramírez, Rafael, Fernández-Portillo, Antonio Díaz-CASero, Juan Carlos (2019). "Green Start-Ups' Attitudes towards Nature When Complying with the Corporate Law!. Complexity, 2019. DOI: $10.1155 / 2019 / 4164853$

Robina Ramírez, Rafael and Suárez, Luis Manuel (2019). "Gestión ética y sostenible del corporate compliance en empresas turísticas. XIX Jornadas Hispanolusas". Osuna, DOI 10.14255/2071-789X.2017/11-3/2

Robina-Ramírez, R. and Merodio, J. A., (2019). "Transforming students' environmental attitudes in schools through external communities". Journal of Cleaner Production, 232, 629-638.

Robina-Ramírez, R.; Merodio, J.A.M., and McCallum, S. (2020). "What role do emotions play in transforming students' environmental behaviour at school?" Journal of Cleaner Production, 258, 120638.

Sarstedt, Marko, Hair, Joseph, Ringle, Christian, Thiele, Kai and GuderGAN, Siegfried (2016). "Estimation issues with PLS and CBSEM: Where the bias lies!". Journal of Business Research, 69(10): 3998-4010.

Setó-Pamies, Dolors and Papaoikonomou, Eleni (2016). "A multi-level perspective for the integration of ethics, corporate social responsibility and sustainability (ECSRS) in management education". Journal of Business Ethics, $136(3): 523-538$. 
Solís, Ángels (2008). "El impacto de la actividad universitaria sobre el medio ambiente". Revista Eureka sobre Enseñanza y Divulgación de las Ciencias, 5(3).

Stone, Mervyn (1974). "Cross-validatory choice and assessment of statistical predictions". Journal of the Royal Statistical Society: Series B (Methodological), 36(2): 111-133.

Tilbury, Daniella (2011). "Higher education for sustainability: a global overview of commitment and progress". Higher education in the world, 4:18-28.

Ull, Ángeles, Martínez, Pilar, Piñero, Albert y Aznar, Pilar (2010). "Análisis de la introducción de la sostenibilidad en la enseñanza superior en Europa: compromisos institucionales y propuestas curriculares". Revista Eureka sobre Enseñanza y Divulgación de las Ciencias, 7: 413-432.

United Nations (UN) (2012). “The future we want". Outcome document of the United Nations Conference on Sustainable Development Rio de Janeiro, Brazil, 20-22 June. [Accessed 3 February 2019] Available at: <http://www. un.org/ga/search/view_doc.asp?symbol=A/RES/66/288\&Lang=E > .

United Nations Economic Commission for Europe (UNECE) (2005). "The UNECE Strategy for Education for Sustainable Development CEP/ AC.13/2005/3/Rev1". UN Economic and Social Council, Paris.

United Nations Educational, Scientific and Cultural Organization (UNESCO) (1997). Thessaloniki Declaration. Thessaloniki, Greece, 1997.

United Nations Educational, Scientific and Cultural Organization (UNESCO) (2005). "United Nations decade of education for sustainable development 2005-2014: International implementation scheme". Paris: UNESCO. [Accessed on 15 November 2017] Available at: <http://unesdoc. unesco.org/images/0014/001486/148654E.pdf>.

United Nations Educational, Scientific and Cultural Organization (UNESCO) (2010). "Education for Sustainable Development Lens: A Policy and Practice Review Tool". Series: Education for Sustainable Development in Action, Learning \& Training Tools No. 2. UNESCO Education Sector, Paris: UNESCO. [Accessed 10 January 2019] Available at: <https:// unesdoc.unesco.org/ark:/48223/pf0000190898>.

United Nations Educational, Scientific and Cultural Organization (UNESCO) (2014). "Roadmap for Implementing the Global Action Programme on Education for Sustainable Development". Paris, France. [Accessed 10 January 2017] Available at <http://unesdoc.unesco.org/ images/0014/001486/148654e.pdf>.

United Nations Educational, Scientific and Cultural Organization (UNESCO) (2015). "Global action programme on education for sustainable 
development information folder". [Accessed 10 January 2019] Available at: $<$ https://en.unesco.org/esd-repo/662/>.

VAre, Paul and Scott, William (2007). "Learning for a change: Exploring the relationship between education and sustainable development". Journal of Education for Sustainable Development, 1(2): 191-198.

Vilches, Amparo y Gir, Daniel (2012)."La educación para la sostenibilidad en la Universidad: el reto de la formación del profesorado". Profesorado. Revista de currículum y formación de profesorado, 16(2): 25-43.

Vilches, Amparo y Gil, Daniel (2007). "La necesaria renovación de la formación del profesorado para una educación científica de calidad”. TED: Tecné, Episteme y Didaxis, (22).

Vincent, Shirley and Will Focht (2009).“US higher education environmental program managers' perspectives on curriculum design and core competencies: Implications for sustainability as a guiding framework". International Journal of Sustainability in Higher Education 10(2): 164-183.

Waas, Tom, Verbruggen, Aviel and Wright, Tarah (2010). "University research for sustainable development: definition and characteristics explored". Journal of cleaner production, 18(7): 629-636.

Waples, Ethan, Antes, Alison, Murphy, Stephen, Connelly, Shane \& Mumford, Michael (2009). "A meta-analytic investigation of business ethics instruction". Journal of Business Ethics, 87(1): 133-151.

Wright, Tarah (2002). "Definitions and frameworks for environmental sustainability in higher education". Higher education policy, 15(2): 105-120.

Wright, Tarah (2004). "The evolution of sustainability declarations in higher education". Higher education and the challenge of sustainability, 7-19. Springer, Dordrecht.

Zarifian, Philippe (1999). Objectif Competente, pour une nouvelle logique. Paris: Éditions Liasions

Zealand, N. (2004.) "Parliamentary Commissioner for the Environment. Farming Trends, in Growing for Good: Intensive Farming, Sustainability and New Zealand's Environment", Wellington: Parliamentary Commissioner for the Environment, 31-53. 